\title{
PHONON-LIKE LIGHT SCATTERING IN POLYCRYSTALLINE CARBON STRUCTURES
}

\author{
A.P. NAUMENKO ${ }^{1}$ N.E. KORNIYENKO ${ }^{1}$ V.M. YASHCHUK, ${ }^{1}$ \\ V.N. BLIZNYUK, ${ }^{2}$ S. SINGAMANENI ${ }^{3}$
}

${ }^{\mathbf{1}}$ Faculty of Physics, Taras Shevchenko National University of Kyiv

(4, Academician Glushkov Prosp., Kyiv 01601, Ukraine; e-mail: a_naumenko@univ. kiev. ua)

${ }^{2}$ Western Michigan University

(Kalamazoo, Michigan 49008, USA)

PACS 78.30.-j, 78.30.LY,

63.200

${ }^{3}$ School of Engineering Applied Sciences, Washington University

(C) 2012

(St. Louis, MO 63130, USA)

A new type of inelastic light scattering in a disordered condensed matter is considered, in which, contrary to the conventional Raman scattering with fixed frequencies of vibrations, a shift of vibrational band positions is observed under variation of the excitation radiation frequency. This phonon-like scattering is inherent to intermediate states of the matter characterized by a "smeared" zone structure at the transition from individual non-correlated molecules of an amorphous material to a highly correlated structural state in single crystals. In particular, for different carbon structures, the intermediate structural states between graphite and diamond include some shares of $s p^{2}$ and $s p^{3}$ hybridizations. A defect band and additional ones ( $\mathrm{D}$ and Ad bands, respectively) have been clearly detected in the Raman spectra of such carbon forms. The bands have a doublet frame with the "redistribution" of intensities between components of the doublet (at 1350 and $1375 \mathrm{~cm}^{-1}$ ) in different structural modifications of graphite sensitive to the local environment of the corresponding chemical groups and to physical factors like pressure, temperature, and ionic implantation. The deconvolution of the composite bands into individual components has been performed through the subtraction spectra calculation. The origin of doublets and limitations of the applicability of a coupled double resonance concept are discussed. To determine the nature of the considered Raman bands, their fine structure and asymmetry are analyzed and compared with the behavior of similar bands in diamond-like hydrocarbon films and carbon metastable phases under high pressure.

\section{Introduction}

Graphite materials are excellent subjects for fundamental investigations directed toward finding the correlations between spectral features and a structural order due to a large number of structural modifications of graphite and a relatively small number of vibrational modes observed in their Raman scattering and IR absorption spectra. From inelastic neutron scattering experiments, one can find a dispersion of phonon branches in the whole Brillouin zone (BZ). However, only the states at the center of $\mathrm{BZ}$ are involved in the processes of first-order Raman scattering and IR absorption. A rather high accuracy of optical measurements allows the detection of features in vibrational spectra usually unobservable by diffraction techniques. In the case of superlattice structures, defects, or fine-dispersed powder samples, the non-centered vibrational modes resulting from phonon branches "folding" may be transformed to the "central" modes $(\mathbf{k} \cong 0)$, thus allowing their registration in the first-order Raman and IR spectra. In the case of small-size domains with a random alignment of the crystalline axis, many vibrational states can be registered with optical spectroscopy techniques because of the violation of selection rules for the quasimomentum due to the strong smearing of their energy band structure. This essentially expands spectroscopic possibilities of optical methods. An essential feature of graphite materials' vibrational spectra is the high intensity of their forbidden bands (i.e., bands forbidden by selection rules for the space group $D_{6 h}^{4}$ of graphite), comparable to or even exceeding the intensity of the fundamental bands $[1,2]$. In this respect, the carbon materials differ from liquids, where the rules of selection for the symmetry point groups of molecules are not fulfilled because of the intermolecular interaction and thermal motion, which results, as a rule, in weak "forbidden" bands [3].

In spite of numerous spectral investigations of graphite materials, a less attention has been paid till now to a detailed study of the shape of spectral bands and their correlations with the corresponding structural features. Due to this fact, we have focused our present work on a detailed study of the spectral shapes of both allowed and forbidden bands. We distinctly revealed the bands with maxima at $1350 \mathrm{~cm}^{-1}$ and $1375 \mathrm{~cm}^{-1}$ in the forbidden spectra of some of carbon materials, by using an 
$\operatorname{Ar}^{+}$-laser $\left(\lambda_{L}=514.5 \mathrm{~nm}\right)$ as an excitation source. The essential dependence of the frequency of bands on the wavelength of the exciting radiation is one of the most interesting peculiarities of light scattering spectroscopy [4-7]. In particular, such a feature of inelastic light scattering was observed in a number of carbon materials, for example in disordered graphite, glassy carbon, and diamond-like and carbon films for fundamental bands at 1350, 1380, 1550, and $1600 \mathrm{~cm}^{-1}\left(\lambda_{L}=514.5 \mathrm{~nm}\right)$ and for some overtones $[4,5]$. The frequency shift of these bands is more than $40-50 \mathrm{~cm}^{-1}$ for a change in the excitation light frequency of $5 \times 10^{3} \mathrm{~cm}^{-1}$. Moreover, in the majority of cases, the frequencies of the observed bands decrease with increase of $\lambda_{L}[6,7]$. At the same time, as a rule, the frequencies of other narrower bands, for example 1582 and $1332 \mathrm{~cm}^{-1}$, are neither displaced nor changed in lower limits. The generalization of experimental and theoretical data concerning this problem allows us to address a new type of scattering of light, namely the phonon-like scattering of light, contrary to the Raman scattering (RS) on molecule vibrations or phonons in perfect crystals. The phonon-like scattering, as a characteristic of disordered phases, is not described by a definite wave vector. In particular, such type of light scattering is a characteristic-like coordination.

\section{Experimental Details}

Because of the strong absorption in graphite at optical frequencies (the absorption coefficient is $2.4-2.6 \mathrm{~cm}^{-1}$ and the skin depth at $\lambda_{L}=514.5 \mathrm{~nm}$ is about $400 \AA$ ), we have used the Brewster backscattering geometry in our experiments. The light source used was a 514.5-nm and 488-nm $\mathrm{Ar}^{+}$-laser with linearly polarized emission. A particular wavelength of an $\mathrm{Ar}^{+}$laser was selected with a prism located outside of a laser resonator, and a cylindrical lens was used to focus light on a $10 \times 0.1 \mathrm{~mm}^{2}$ spot. It is worth mentioning that the larger illuminated area can efficiently eliminate the local heterogeneity (more reliable data) and minimize the probability of radiation damage of the sample due to the resonance adsorption. The spectra were detected by an automated double spectrometer DFS-24 (LOMO, Russia) equipped with a cooled photomultiplier and a registration system working in the photon counting mode. In connection to the numerical analysis, the spectra were digitalized in a wide frequency range with a fixed increment (from $1 \mathrm{~cm}^{-1}$ up to $5 \mathrm{~cm}^{-1}$ ). Because of the relatively small cross-section of Raman scattering (RS) in graphite materials and in order to obtain a more reliable information, an additional noise minimization technique was applied.
In particular, the relatively wide spectral windows of $3-$ $5 \mathrm{~cm}^{-1}$, long acquisition time, and optimized digital averaging of spectra with a variable spectral window were used for the weak signal amplification.

Single crystals of highly oriented pyrolytic graphite (HOPG), polycrystalline anisotropic and isotropic samples with various crystallite sizes $L_{a}$ (from 200 to $1000 \AA$ ), flakes of natural graphite, samples of thermoexpanded graphite pressed at a pressure of $200 \mathrm{~atm}$ were investigated. In the case of anisotropic samples of single- or polycrystals, the RS spectra were collected under reflection from a fresh cleavage (along the crystalline planes) or from the sample edge, as a surface being perpendicular to the graphite planes [8]. Both spectra from the graphite plane and from the edges of a sample were studied. In the latter case, the exciting radiation has been polarized in a plane containing the optical axis $c$. Such a configuration is the most sensitive to out-of-plane vibration modes.

\section{Results and Discussion}

\subsection{Doublet structure of forbidden bands and the phenomena of the distribution of intensities}

Because of their strong sensitivity to the structure of materials, the studies of the forbidden bands may be more informative than the traditional investigations of the allowed band $\mathrm{E}_{2 g}$ [1]. Typical Raman spectra of investigated graphite materials at different excitation wavelengths are shown in Figs. 1 and 2. We have carried out experimental investigations and comparative analysis of spectra in the range of the allowed $\mathrm{E}_{2 g}$ G-band of graphite $\left(1500-1650 \mathrm{~cm}^{-1}\right)$ and in the region of the forbidden D-band $\left(1310-1410 \mathrm{~cm}^{-1}\right)$. All spectra were normalized to the maximum of intensity after the background subtraction. Some of the spectra are shifted along the $Y$-direction for clarity with an indication of the corresponding base lines. First of all, one can see that, in HOPG, a sharp Raman band with the maximum at $1580 \mathrm{~cm}^{-1}$ and the half-width of about $10 \mathrm{~cm}^{-1}$ is observed (Fig. 1). This band corresponds to the Raman active mode $\mathrm{E}_{2 g}$ at the center of BZ, which is in agreement with [8]. The same narrow spectral band is also observed from the edges of graphite layers. These observations are in good agreement with [9]. From Figs. 1 and 2, one can also observe that, with increasing a degree of disorder, the allowed bands $\mathrm{E}_{2 g}$ broaden, "move" to the high-frequency region by $5-7 \mathrm{~cm}^{-1}$ and cause a well-pronounced asymmetry on the high frequency 

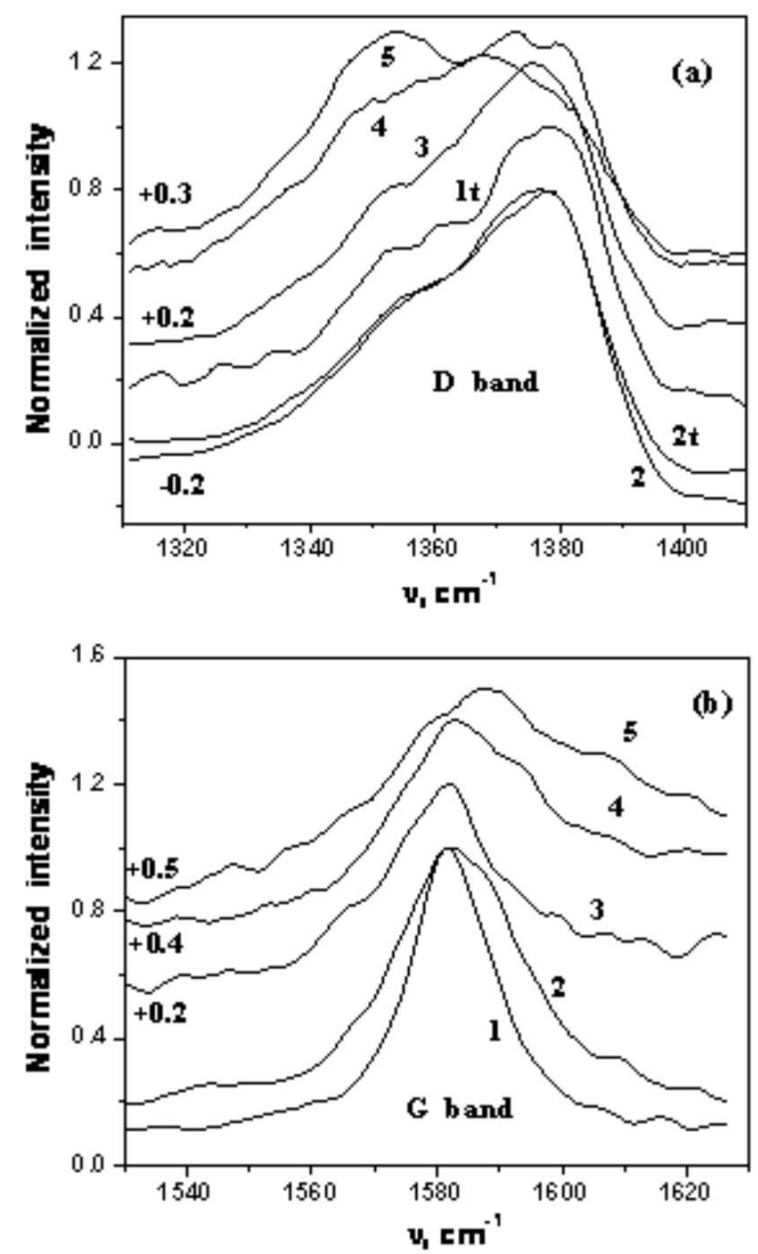

Fig. 1. Raman spectra of various forms of graphitic carbon in the region of "forbidden" D $(a)$ and graphite G $(b)$ bands normalized to the maximum of the intensity recorded under excitation with a wavelength of $514.5 \mathrm{~nm}$ for: (1) single crystal of highly oriented pyrolythic graphite (HOPG), (2) anisotropic graphite with the size of crystallites $L_{a} \approx 300 \AA$, (3) natural graphite, isotropic graphite with the crystallite size of $L_{a} \approx 10^{3} \AA$, (4) and $200 \AA$ (5). Symbol $t$ shows Raman $\mathrm{D}$ bands registered under light scattering from the edges of corresponding samples. Some spectra are shifted along the vertical axis for clarity and corresponding values are indicated on the figure

side. For $\lambda_{L}=488 \mathrm{~nm}$, a well-distinguished band at $1625 \mathrm{~cm}^{-1}$ appears on the high-frequency wing of the $\mathrm{E}_{2 g}$-band, while being practically not observable under the $\lambda_{L}=514.5 \mathrm{~nm}$ excitation.

The most significant changes of Raman spectra are observed in the forbidden bands near $1310-1410 \mathrm{~cm}^{-1}$. For HOPG according to the selection rules, these bands should not be observed. However, in samples of anisotropic graphite with the crystallite size $L_{a}=300 \AA$,
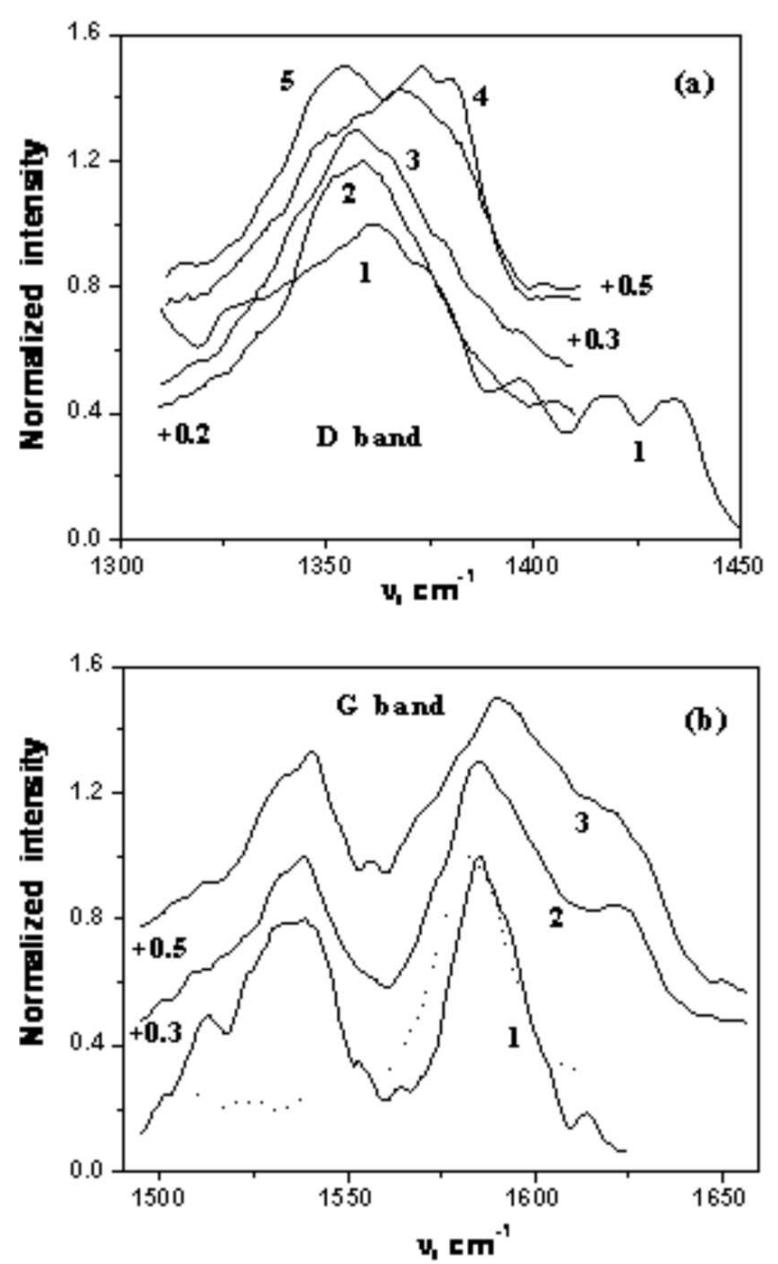

Fig. 2. Raman spectra of various graphite modifications in the region of $\mathrm{D}(a)$ and graphite $\mathrm{G}(b)$ bands normalized to the maximum of the intensity recorded under excitation with a wavelength of $488 \mathrm{~nm}$ for: anisotropic graphite with the size of crystallites $L_{a} \approx 300 \AA$ (curve 1 ), isotropic graphite with the crystallite size $L_{a} \approx 10^{3} \AA$ (curve 2), and $L_{a} \approx 200 \AA$ (curve 3). D bands of graphite samples with smaller (curve 4) and greater (curve 5) crystallite sizes $(a)$, as well as the $\mathrm{G}$ band of anisotropic graphite (b, dashed line) recorded under the 514.5-nm excitation, are also shown for comparison. Some spectra are shifted along the vertical axis for clarity and corresponding values of the shift are indicated

these bands have intensities comparable with the intensity of the allowed band. For the majority of samples under study, the forbidden band intensities are generally higher than the intensity of the fundamental band $\mathrm{E}_{2 g}$. This seemingly paradoxical situation may be natural in graphite and can be explained by the high density of vibrational states (DOS) at $\mathrm{K}$ and $\mathrm{M}$ points on the border of BZ [1]. A similar situation is also observed in the Raman spectra of a high-temperature superconduc- 
tor $\mathrm{YBa}_{2} \mathrm{Cu}_{3} \mathrm{O}_{x}(6<x<7)$ under the intensive pulse excitation $\left(38 \mathrm{~kW} / \mathrm{cm}^{2}\right.$ with $\lambda_{L}=488$ and $\left.514.5 \mathrm{~nm}\right)$ [18]. However, when the laser beam intensity is lowered to a few $\mathrm{kW} / \mathrm{cm}^{2}$ or when the temperature is lower than $80^{\circ} \mathrm{K}$, the intensity of the forbidden bands in this superconductor also essentially decreases.

A weak resolved doublet at $1350-1370 \mathrm{~cm}^{-1}$ in the Raman spectra of disordered graphite was reported. However, its structure has not been investigated in detail neither in [4] nor in subsequent papers [7-13] and so has not received an appropriate physical interpretation. The detailed investigation of the shapes of the forbidden vibrational bands in various graphite materials at $\lambda_{L}=514.5 \mathrm{~nm}$ (part of results is shown on Fig. 1) has allowed their decomposition into bands with maxima at $1350 \mathrm{~cm}^{-1}$ and $1375 \mathrm{~cm}^{-1}$ and the observation of the Raman intensity redistribution between these forbidden bands. This phenomenon is clearly manifested by a variation of the shape of the envelope band observed in different structural forms of graphite under the same experimental conditions. In particular, for the anisotropic and several isotropic graphite samples with the average domain size of $L_{a}=200-1000 \AA$, the most intensive band is the one at $\nu_{\max }=1350 \mathrm{~cm}^{-1}$. While in other misaligned graphite samples (especially in the case of reflection of RS radiation from the edge), the band at $1375 \mathrm{~cm}^{-1}$ is more intensive. Contrary to the G-band behavior, the observed D-line is not sensitive to the degree of grain misalignment. Previously, these bands were observed individually in different types of diamond-like amorphous films and glassy carbons depending on the excitation wavelength $\lambda_{L}$. In the case of $\lambda_{L}=514.5 \mathrm{~nm}$, there was a possibility to distinguish between vibration bands at $1380 \mathrm{~cm}^{-1}$ and $1540 \mathrm{~cm}^{-1}$ in amorphous films and $1350 \mathrm{~cm}^{-1}$ and $1590 \mathrm{~cm}^{-1}$ in glassy carbons [6]. However, we have presented a more detailed mathematical analysis of the spectra with the decomposition of the bands of diamond-like films [15] into two (as in [6]) and more constituent lines with a possibility of the variation of their individual position, intensity, shape, and width. The experimental Raman bands were separated (a sharp peak over relatively broad band) into two components at 1347 and $1375 \mathrm{~cm}^{-1}$ as the most realistic scenario. One should also note that similar lines along with the intensive band at $1332 \mathrm{~cm}^{-1}$ are often observed in Raman spectra of a crystalline diamond doped with boron [15].

The most significant feature observed in spectra recorded with $\lambda_{L}=488 \mathrm{~nm}$ is the appearance of an additional relatively strong band (Ad-band) located near the $\mathrm{G}$ band at $1540 \mathrm{~cm}^{-1}$ as shown in Fig. 2,b. This band also has a doublet structure with a shoulder observed on the higher frequency side $\left(1510 \mathrm{~cm}^{-1}\right)$. In the case of $\lambda_{L}=514.5 \mathrm{~nm}$ due to a low overall intensity of the lines, only a small signature of these bands can be seen in the second derivative spectra (lower frequency side of a wide $\mathrm{E}_{2 g}$ band of the subtraction spectra is discussed in the next section of this paper). Similar bands (i.e., tunable with the variation of an incident wavelength $\lambda_{L}$ ) but without a well-pronounced doublet structure were reported for diamond-like films [6]. In amorphous carbon without annealing, such variation of the $\mathrm{G}$ band position was observed in the range of 1500 to $1600 \mathrm{~cm}^{-1}$ [9]. We believe that, as in the latter case, the assignment of the bands to the $\mathrm{G}$ or Ad band was ambiguous. In polycrystalline diamond films with a sharp peak at $1332 \mathrm{~cm}^{-1}$, the additional broad band near $1550 \mathrm{~cm}^{-1}$ was also observed and ascribed to the amorphous carbon [9]. In carbon and diamond-like films, simultaneously two forbidden bands D and Ad can be observed despite the fact of their significant overlap [17]. Various physical stimuli have been applied simultaneously with Raman spectroscopy experiments in order to reveal the nature of the observed bands. As a result of the carbon film exposure to a beam of $\mathrm{Ar}^{+}$ions, the $1366-\mathrm{cm}^{-1}$ band is shifted to $1382 \mathrm{~cm}^{-1}$, and the band at $1571 \mathrm{~cm}^{-1}$ to $1520 \mathrm{~cm}^{-1}$. Under the bombardment of the same films with $\mathrm{Xe}^{-}$ ions, the $\mathrm{D}$ band is observed at $1380 \mathrm{~cm}^{-1}$. Under the $\mathrm{Xe}^{-}$bombardment, the Ad band is shifted from $1508 \mathrm{~cm}^{-1}$ to $1520 \mathrm{~cm}^{-1}[6,17]$. Under the annealing of amorphous carbon at $600{ }^{\circ} \mathrm{C}$, the $\lambda_{L}$-dependent Ad band becomes narrower and is stabilized at $1600 \mathrm{~cm}^{-1}$, probably being transformed into the G band [9]. Similarly, in the range corresponding to the position of the $\mathrm{D}, \mathrm{Ad}$, and $\mathrm{G}$ bands under appropriate resonance conditions, we have observed a doublet structure with the corresponding frequencies $1350-1375 \mathrm{~cm}^{-1}, 1510-1540 \mathrm{~cm}^{-1}$, and $1590-1626 \mathrm{~cm}^{-1}$. It is significant that the frequencies of the doublet components of the D and Ad bands remain practically unchanged, contrary to the $\mathrm{E}_{2 g}$ band behavior as illustrated in Figs. 1 and 2 .

\subsection{Origin of the doublet structure of vibration bands of graphite materials}

The existence of the doublet vibration bands was reported for graphite materials in many publications. For example, the high resolution spectra of the $G$ band $\left(1580 \mathrm{~cm}^{-1}\right)$ typically show a high-frequency shoulder at $1620 \mathrm{~cm}^{-1}$, which is particularly well observed under $\lambda_{L}=488 \mathrm{~nm}$ (see Fig. 1) $[4,8,11,16]$. In the overtone region of quasicrystalline graphite, a doublet band 2695-2735 $\mathrm{cm}^{-1}$ [4] (also at 2697-2739 $\mathrm{cm}^{-1}$ [5]) or a 
band with a low-frequency shoulder near $2735 \mathrm{~cm}^{-1}$ has been reported $[11,16]$. In the same range of the spectra of glassy carbon, an intensive single line at $2720 \mathrm{~cm}^{-1}$ is observed, the frequency of which is strictly correlated with the position of the $\mathrm{D}$ band at $1360 \mathrm{~cm}^{-1}$ under variation of the crystalline size [5]. This observation confirms the interpretation of the $2720-\mathrm{cm}^{-1}$ band as an overtone of the $\mathrm{D}$ band. Interestingly, a doublet overtone band (components at 2697 and $2739 \mathrm{~cm}^{-1}$ at $\lambda_{L}=488$ $\mathrm{nm}$ ) reported in [5] is, in fact, a manifestation of overtones of the components of the D band at $1350 \mathrm{~cm}^{-1}$ and $1375 \mathrm{~cm}^{-1}$ (also observed by us) and which can be tuned significantly with a variation of $\lambda_{L}$. The existence of the doublet structure and its frequency dependence have been explained in [5] through a coupled double resonance (CDR) concept. In accordance to this approach, the appearance of this doublet is caused by a splitting of the electronic $\pi$ zone of $3 \mathrm{D}$ graphite due to interactions between neighboring graphite sheets. As a result, there are two wave vectors (for excited electrons $\mathbf{k}$ and for excited phonons q) corresponding to resonance laser photons $\eta \omega_{L}$ which results in the appearance of two vibration frequencies corresponding to two known dispersion bands. However, as pointed in [5], the conditions of CDR are satisfied only for the phonon wave vectors larger than those of photons. Therefore, the effect can be observed in RS spectra of the first order only. In accordance to such a consideration, the existence of a forbidden $\mathrm{D}$ band in the fundamental frequency range reported in $[4,5]$ due to vibration states near $\mathrm{K}$ point of the Brillouin zone (in the KM direction) for highly disordered boundary regions of crystalline domains is possible. In these disordered regions, the graphite sheets are practically decoupled, and the dispersion equations for a single graphite layer can be used as a good approximation for the D band analysis [5]. Naturally, the D band and its overtone appear as a single line in this case (which is supported both by the theoretical consideration and by experimental observations [5]). This is the reason behind the doublet structure of the forbidden D band observed by us, not being explained in the framework of the CDR model. Nevertheless, the effect can find a reasonable explanation through closely located dispersion bands or the same phonon dispersion band considered at different points of BZ. In accordance to $[11,12]$, such a situation is realized for a weak $2450-\mathrm{cm}^{-1}$ band having two components at $2435 \mathrm{~cm}^{-1}$ and $2468 \mathrm{~cm}^{-1}$ interpreted as the combination tones of the fundamental bands at 830, 850, and $1620 \mathrm{~cm}^{-1}$.

The doublet structure of the $\mathrm{D}$ band $\left(\lambda_{L}=514.5 \mathrm{~nm}\right)$ has been observed in the spectra of all carbonaceous ma- terials under study. Thus, for $\lambda_{L}=488.0 \mathrm{~nm}$, the D band with maxima near $1357 \mathrm{~cm}^{-1}$ was practically simple, which is illustrated in Fig. 1,c, where the doublet D bands excited by $\lambda_{L}=514.5 \mathrm{~nm}$ were given for comparison. One can see that these single bands correspond to the low-frequency component with $1350 \mathrm{~cm}^{-1}$. A small high-frequency shift of the D band under excitation at $\lambda_{L}=488.0 \mathrm{~cm}^{-1}$ is connected with a regular frequency shift, when $\lambda_{L}$ is changed [4-6]. However, this shift is essentially smaller than the frequency interval of the observed doublet. Summarizing the above discussion, one can say that the doublet structure of the D, Ad, and G bands observed by us is probably associated both with the peculiarities of a structure of carbonaceous materials and with the resonance conditions of excitation of the Raman spectra. The fact of the existence of the overtone doublet structure in the RS spectra of graphite single crystals and the observation of three components in the overtone Raman spectra of hydrocarbon films [7], which are connected with a doublet structure in the fundamental region and the observation of the doublet structure 1350-1375 $\mathrm{cm}^{-1}$ in diamond-like films and even single diamond crystals [15], emphasizes the need for the additional analysis of the CDR conception and a more detailed explanation of the origin of the doublet band structure.

\subsection{Subtraction spectra analysis}

The phenomena of the intensity redistribution of forbidden peaks, which is observed as a change of line shapes in various structural forms of graphite, can be used for identifying the components of observed doublets. An approximate determination of the shape and the position of these constituting peaks can be done via the mathematical procedure of subtraction of the corresponding normalized spectra. The spectra were normalized to their maximal intensity after the preliminary subtraction of the background. Some of the subtraction spectra obtained under such a procedure are shown in Fig. 3.

In particular, the plot illustrates the extraction of the high frequency component (Fig. 3,a) and the lowfrequency one (Fig. 3,b). One can see that the low frequency component is broader and has some lowfrequency shift from the original position $1350 \mathrm{~cm}^{-1}$. This fact can be identified by the existence of the third low-frequency component of the forbidden band. As a result, the subtracted bands in Fig. 3,b have broader shape and are shifted due to a combined contribution from two low-frequency components. Figure 3, $a$ shows the subtraction spectra of the $\mathrm{G}$ band of isotropic graphite 

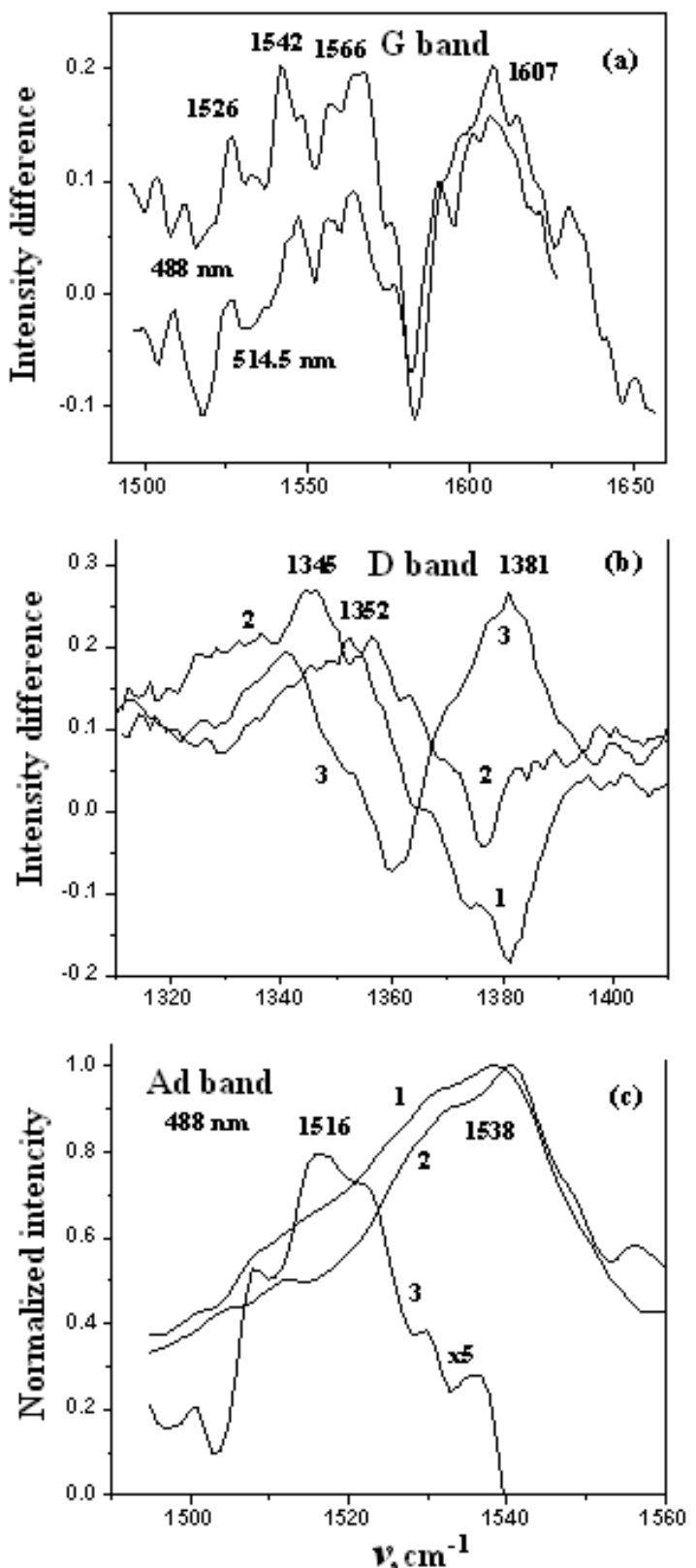

Fig. 3. G band (a), D band (b), and Ad band (c) portions of differential Raman spectra obtained by the subtraction of normalized spectra of various graphite materials: $(a)$ two isotropic graphite samples with crystallite size $L_{a}$ of 200 and $1000 \AA\left(\lambda_{L}=488 \mathrm{~nm}\right.$ and $\left.\lambda_{L}=514.5\right)$; (b) two isotropic graphite samples $\left(L_{a} \approx 200\right.$ $1000 \AA$ ) recorded under excitation with $\lambda_{L}=514.5 \mathrm{~nm}$ (curve 1), isotropic and natural graphite (both for $514.5 \mathrm{~nm}$ ) (curve 2), and two isotropic graphite samples (200-1000 $\AA$ crystallite size) for excitation with $\lambda_{L}=514.5$ and $488 \mathrm{~nm}$, correspondingly (curve 3); (c) normalized spectra of isotropic graphite $\left(L_{a} \approx 200 \AA\right)$ (curve 1), anisotropic graphite $\left(L_{a} \approx 300 \AA\right.$ ) (curve 2), and their differential spectrum magnified 5 times (curve 3) recorded at $\lambda_{L}=488 \mathrm{~nm}$ with various sizes of crystallites $L_{a}$. In accordance to the existing dispersion data for crystalline graphite, the maximum of the dispersion band of $\mathrm{E}_{2 g}$ mode coincides with the center of BZ, which should appear in a pronounced asymmetry of the band versus the frequency and its bathochromic shift. In Fig. 3,a, the results of subtraction of G-bands of isotropic graphite characterized by crystallite size $L_{a}$ are shown. As $L_{a}$ decreases, the spectral bands in the G-band region should decrease because of the selection rules and the dimensional disclosing of the vibrational zone $[5,16]$. It is well known that the maximum of the dispersion branch of $\mathrm{E}_{2 g}$ mode is located at the center of BZ and manifests itself by an asymmetry of the band and a shift to the lowfrequency region. However, as was discussed above, a high-frequency asymmetry was experimentally observed. This fact is explained by the relaxation of selection rules and the simultaneous presence of dipole-active $\mathrm{E}_{1 u}$-mode $\left(1588 \mathrm{~cm}^{-1}\right)$ together with allowed $\left(\mathrm{E}_{2 g}\right)_{2}$-mode. For an isotropic sample with $L_{a}=200 \AA$, the maximum of the Raman band is observed at $\nu_{\max }=1587 \mathrm{~cm}^{-1}$, which is very close to the frequency of $\mathrm{E}_{1 u}$ mode. The subtraction spectra in a vicinity of the $\mathrm{G}$ band display the vibrational states $1600 \mathrm{~cm}^{-1}$ and a wide spectrum of lowfrequency vibrations. The high-frequency subtraction band is caused by the states of $\mathrm{E}_{1 u}$ mode located near the minimum and the maximum of the dispersion branch placed at the center of BZ (point $\Gamma$ ) and at the point $\Sigma$ along the $\Gamma-K$ direction, respectively $[1,16]$. Note that the states close to the maximum of the $\mathrm{E}_{1 u}$ branch (point $\Sigma$ ) are displayed as a shoulder at $1625 \mathrm{~cm}^{-1}$ of the $\mathrm{G}$ band (see Fig. 3,a) and as an overtone band at $3248 \mathrm{~cm}^{-1}[11,16]$.

The low-frequency subtractive band corresponds to the additional Ad band. The subtractive band observed in the region of the additional Ad band is shown in Fig. $3, c$ and corresponds to a low-frequency component of the second doublet.

\subsection{Analysis of the asymmetry and fine band structure}

The easiest way to determine the origin of the forbidden bands at 1350,1375 , and $1540 \mathrm{~cm}^{-1}$ is based on the consideration of their phonon dispersion curves in a graphite single crystal along the directions of high symmetry $[1,16]$. The band $1375 \mathrm{~cm}^{-1}$ can be described as that corresponding to the longitudinal acoustic (LA) mode at $\mathrm{M}$ point of BZ of graphite [1]. The frequency of the band at $1350 \mathrm{~cm}^{-1}$ is very close to the frequency of "merged" two optical $\left(\mathrm{E}_{1 u}, \mathrm{E}_{2 g}\right)$ and one acoustical (LA) 
modes at $\mathrm{K}$ point of BZ. The existence of the Ad band at $1540 \mathrm{~cm}^{-1}$ can be explained as a state of the $\mathrm{E}_{1 u}$-mode near $\mathrm{M}$ point $[1,16]$. These vibrational states on the edge of the Brillouin zone can appear in the first-order Raman spectra, even when these vibrational states are folded on the center of BZ. This remains possible in the case of the formation of a polytypic structure or structural disorder. The high intensities of bands in the region of $1350-1375 \mathrm{~cm}^{-1}$ and $1510-1540 \mathrm{~cm}^{-1}$ can be explained by a high density of available vibrational states (DOS) in the corresponding areas in comparison with those of the $\left(\mathrm{E}_{2 g}\right)_{2}$-mode at the center of $\mathrm{BZ}$.

The existence of a sharp edge of the band and its asymmetry may be important for the correct interpretation of vibration spectra. For instance, the band at $3250 \mathrm{~cm}^{-1}$ observed in [16] has a high-frequency edge, which is in agreement with its common interpretation as an overtone of the $1625 \mathrm{~cm}^{-1}$ vibration state of mode $\mathrm{E}_{1 u}$ near the maximum at $\Sigma$ point of the dispersion curve along the $\Gamma \mathrm{K}$ axis of BZ [1]. On contrary, the band at $2450 \mathrm{~cm}^{-1}$ has a low-frequency edge [16]. Hence, this vibration should correspond to the minimum of the phonon dispersion curve. Therefore, they cannot be considered as the components of 830, 850, and $1620 \mathrm{~cm}^{-1}$ vibrations, as considered in [11], because each of the latter vibration states corresponds to the maximum of the dispersion curve. More likely, this band is located near the bottom of the zone near $1225 \mathrm{~cm}^{-1}$ frequency. The vibrational state, which is close in frequency and corresponds to the transverse acoustic (TA) branch at $\mathrm{K}$ point, cannot be assigned to this vibration (or at least it cannot contribute considerably to this vibration state) because of its location on the top of the zone. The actual vibration state is, probably, the state of mode $\mathrm{E}_{1 u}$ near a deep minimum of the dispersion curve at $\mathrm{K}$ point of BZ. In favor of such assignment is a one-side-smeared asymmetric peak observed at $2450 \mathrm{~cm}^{-1}$ in graphite samples with the average domain size of $1 \mu \mathrm{m}$ and even HOPG [16]. It worth mentioning that there is a considerable discrepancy of literature data concerning the behavior of dispersion curves of $\mathrm{E}_{1 u}$ mode near the minimum at $\mathrm{K}$ point. We note also that, in comparison to a broader $2450 \mathrm{~cm}^{-1}$ band, the one at $3250 \mathrm{~cm}^{-1}$ is much narrower for the same sample, and its one-side broadening is observed only in samples with much more disordered crystallites with a size of $25 \AA$. The difference in the broadening behaviors of these bands is due to the uncertain nature of the quasimomentum conservation law here. It shows also a smooth or sharp $\omega(k)$ dispersion near the extremes of the dispersion curve. The above arguments can be used for a more precise estimation of the phonon dispersion.

The observed fine structure of the 1350- and $1375-\mathrm{cm}^{-1}$ doublet components can be also be used for interpreting the bands. In particular, for highly dispersed graphite materials (compressed samples of thermally expanded small-grain or colloidal graphite) and anisotropic graphite under light scattering from the edges, each of the 1350 - and $1375-\mathrm{cm}^{-1}$ bands is constituted with two elementary components (1350, $1358 \mathrm{~cm}^{-1}$ and $1370,1379 \mathrm{~cm}^{-1}$ ) in accordance to the second derivative spectra. The singlet structure of the second derivative spectra gives a hint of some regularity in the spectrum component positions constituting the broad vibration band. These observations support the idea of universality of the structure formation in dispersed graphite materials. The important things here are that the high-frequency component is more intense for the $1375-\mathrm{cm}^{-1}$ band, while the low-frequency component is more intense for the $1350-\mathrm{cm}^{-1}$ band. This interpretation is confirmed by the fine structure of the 1350-1375- $\mathrm{cm}^{-1}$ doublet. If we consider the secondary doublet structure to be caused by some physical factors (such as polytypic), the observed intensity correlation of the fine structure should confirm the dominant contribution from the LA mode to the $1375-\mathrm{cm}^{-1}$ band. In the case of a considerable contribution to the $1350-\mathrm{cm}^{-1}$ band from the states located close to the minimum of the optical modes, the low-frequency component should dictate its fine structure, which is in agreement with the observed spectra. The considerable contribution to the $1350-\mathrm{cm}^{-1}$ band originates from the states close to the minimum of the optical modes near point $\mathrm{K}$ of BZ.

However, such interpretation may be not exhaustive. One should take several more arguments into account. Firstly, the DOS maximum should be "tethered" to some particular frequency while the observed spectral bands are frequency shifted, depending on the excitation wavelength. Secondly, the bands with wavenumbers of 1350 and $1370 \mathrm{~cm}^{-1}$ have been observed in the IR spectra of diamonds $[18,19]$. The $1350-\mathrm{cm}^{-1}$ band corresponds to a separate nitrogen impurity of the substitution type. In this case, according to an electron spin resonance data, an extra-electron is located on one of the $\mathrm{C}=\mathrm{C}$ chemical bonds, extending its length up to $11 \%$. As a result, the symmetry is decreased at least down to $C_{3 v}$, when all vibrational states are allowed in IR and RS spectra. The band at $1370 \mathrm{~cm}^{-1}$ corresponds to aggregations of nitrogen plates in plane 100. A decrease in the linear sizes of graphite plates leads to an increase of the IR bands frequency to $1375 \mathrm{~cm}^{-1}$, when the size reaches $200 \AA$ [1]. In 
some polycrystalline diamond films, an intensive band at $1332 \mathrm{~cm}^{-1}$ and a band at $1550 \mathrm{~cm}^{-1}$ have been observed simultaneously. The band at $1550 \mathrm{~cm}^{-1}$ may have the same origin as "our" Ad band [9]. Its intensity decreases with the increase of the excitation light frequency. Moreover, diamond-like samples synthesized from carbon vapor are characterized with a sharp diamond band at $1330 \mathrm{~cm}^{-1}$ [5-7]. In addition, the wide bands with variable maximum in the limits of $1440-1550 \mathrm{~cm}^{-1}$ are typically observed, although such states do not exist in the DOS of amorphous diamond [20]. Additionally, a sharp peak at $1375 \mathrm{~cm}^{-1}$ can be found in samples of diamondlike films with impurities [15]. All these results show that the observed bands cannot be interpreted as those resulting from the DOS maximum: the existence of the remaining vibrational state zone structure cannot be explained by the dispersion branch alone, but rather by the existence of wide bands which should be taken into account.

\subsection{Origin of the observed bands and their frequency shift}

In accordance to the CDR concept, the second-order Raman spectra may be characterized with spectral bands (not connected to DOS), whose positions are determined by dispersion branches of the electronic or vibrational zone structure and, under strong phonon dispersion conditions, may depend on the frequency (or wavelength) of the excitation light $\lambda_{L}$. This approach explains the variation of the frequency of overtone vibration bands in graphite single crystals under $\lambda_{L}$ [5]. However, this interpretation is less straightforward in application to disordered materials (graphite or diamond like) due to the observed doublet structure of forbidden bands in the range of fundamental mode frequencies. One can name several reasons why the CDR concept cannot be used in the latter case. As mentioned above, the CDR concept is valid for a direct application to the first-order Raman spectra in the fundamental region and also for highly disordered phases for those zone structures, in which vibrational and electronic states are considerably smeared. Secondly, as reported in [6] for diamond-like films, the vibration states tunable with $\lambda_{L}$, which have corresponding frequencies of $1380 \mathrm{~cm}^{-1}$ and $1540 \mathrm{~cm}^{-1}$ at $\lambda_{L}=514.5 \mathrm{~nm}$, were observed. For glassy carbons, the corresponding bands tunable with $\lambda_{L}$ were observed at $1350 \mathrm{~cm}^{-1}$ and $1580 \mathrm{~cm}^{-1}$. Therefore, the bands at $1350 \mathrm{~cm}^{-1}$ and $1380 \mathrm{~cm}^{-1}$ with frequencies close to D-band doublets observed by us appeared separately in different carbon dispersion data based on the CDR. The bands' shift observed under conditions of ionic bombardment provides, in our view, an evidence that they originate from the structural peculiarities of the matter and not from the kinetics of the RS process [17]. Thirdly, the observed overtones at $2697 \mathrm{~cm}^{-1}$ and $2739 \mathrm{~cm}^{-1}$ in graphite single crystals correspond to the fundamental states of $1347.5 \mathrm{~cm}^{-1}$ and $1369.5 \mathrm{~cm}^{-1}$, if we take the observed zero anharmonicity of a glassy carbon into account [5]. In consideration of the same difference in $\lambda_{L}$, the deviation from the doublets components' positions observed by us is negligible. Moreover, the observed value of this doublet splitting is 3 to 4 times smaller than the result of theoretical calculations reported in [5]. However, the usage of dispersion relations for singlelayer graphite in highly disordered regions does not lead to a doublet splitting [5]. Depending on the heat treatment, the components with a frequency lower or similar to $1350 \mathrm{~cm}^{-1}$ or higher than $1370 \mathrm{~cm}^{-1}$ are observed near the $\mathrm{D}$ band position in carbon films [7]. These frequencies are also probably related to the structural peculiarities of the matter. Importantly, the half-width $\delta \nu$ of the above-mentioned low-frequency components varies with $\lambda_{L}$ in the opposite way. In the initial film, $\delta \nu$ goes down with $\lambda_{L}$ for $\nu<1350 \mathrm{~cm}^{-1}$, while $\delta \nu$ increases with $\lambda_{L}$ in an annealed film for $\nu<1370 \mathrm{~cm}^{-1}$ (the latter fact may justify for their different origin) [7].

All the above factors demonstrate that the CDR concept cannot explain all known experimental results and therefore needs a generalization and a development for disordered media. Due to the absence of the long-range order in disordered (amorphous) materials, they cannot be characterized with a particular momentum of the vibrational excitation. One of the existing concepts of the violation of selection rules in the RS process is based on the wave vector shift consideration: $D \mathbf{k}=\mathbf{k}_{L}-\mathbf{k}_{S}-\mathbf{q}=0$, where $\mathbf{k}_{L}$ and $\mathbf{k}_{S}$ are the wave vectors of the incident and scattered (Stokes) radiation, $\mathbf{q}$ is the wave vector of a phonon. The concept introduces a correlation length $\Lambda$, which characterizes the spatial distribution of the normal mode. Under such approach, $q$ is not a proper parameter of the normal mode, and a phase shift $\Delta k=2 \pi / \Lambda$ is allowed. In amorphous media in accordance to Shuker and Gamone, the normal vibration modes and the spatial time correlation are proportional to $e^{\mathbf{q r}} e^{-r / \Lambda}[1]$. However, in the majority of publications on the vibrational states of disordered materials, all vibration states are considered to be localized in accordance to DOS [5]. For many intermediate states of matter between the extremes of the long-range order and the random arrangement of atoms, the vibration modes are delocalized in some spatial region $\Lambda$ having 
wave properties and dispersion bands with the width increasing under reduction of $\Lambda$. In the case of the presence of a residue of the existing zone structure of vibrational states, which is characterized with broad energy bands rather than dispersion curves $\omega(q)$, it is possible to talk in terms of phonon-like excitations and to consider peculiarities of light scattering on them. The distinguished feature of light scattering on such states is the possibility of a considerable variation (tuning) of the frequency of spectral bands in the range of considerable widths of energy dispersion bands. In this connection, we prefer to use a special term of phonon-like light scattering to underline the difference of the process from the traditional Raman scattering, where the frequencies of the bands are fixed. A collective zone structure of a crystal is, first of all, deviated near the boundary of BZ, which is manifested by a weakening of the overtone $2720 \mathrm{~cm}^{-1}$ and its practically disappearance with increase of the structural disorder. This is connected with a small wavelength for states near the BZ boundary. At the center of BZ, due to longer wavelengths of the corresponding excitations, the zone structure may be preserved probably for a longer time.

In the consideration of the phonon-like scattering of light, one should consider a possibility of the existence of various metastable structural states of carbon arising, in particular, under the application of a high pressure $[3,4]$. Under a pressure $P>15 \mathrm{GPa}$, the $\mathrm{G}$ band became broader. Carbon samples loose their metal shine due to the development of a broader band gap structure. Under a pressure exceeding $35 \mathrm{GPa}$, a new "hybrid" state of carbon can be found due to the amorphization of the matter. A hysteresis can be observed in cycles with increasing and decreasing pressure. Simultaneously, the optical transmittance of carbon samples arises, and they became transparent in the visible range of light, when the pressure is increasing to $35-55 \mathrm{GPa}$, while the reduction of the pressure to $P=5 \mathrm{GPa}$ is necessary for their transformation back to the non-transparent state. The disappearance of the vibration band at $3340 \mathrm{~cm}^{-1}$ corresponding to the maximum of the $\mathrm{E}_{1 u}$-mode at $\Sigma$ point in the overtone region of the spectra with the increase of the pressure has been also observed [6]. The authors explained this result by a shift of this maximum to the center of BZ due to a weakening of the coupling of second neighbors, which can lead to a gaufre transformation of the graphite layers before the formation of the amorphous phase with $s p^{3}$-bonding [5]. The broadening of the $\mathrm{G}$ band under high pressure conditions happens mainly due to an increase of the intensity of scattered light in the low-frequency side, where the contribution from $s p^{3}$ bonds is more significant. In accordance to [1], nearly $15 \%$ of $s p^{3}$ bonds are observed in amorphous carbon. Note also that the intensity of the $1375-\mathrm{cm}^{-1}$ band is higher in the Raman spectra recorded from the sample edge (Fig. 2). That is, first of all, true for the atoms on the border of the graphite layers, where the "free" (unemployed) bonds of carbon atoms can "couple" to the neighboring layers forming electronic configurations similar to the tetrahedral $s p^{3}$ hybridization. The existence of the $s p^{3}$ hybridization in disordered carbon materials is confirmed by several other facts noticed before. At first, we should note a monotonous increase of the D-band intensity with the degree of disorder [11-13]. Unlike the behavior of other bands in the region of the fundamental bands, overtones, and combination tones show the increasing intensity with the degree of disorder, which is followed by the decreasing intensity caused by the disappearance of the zone structure. The existence of $s p^{3}$ hybridization has also been confirmed by studies of the radial distribution of the electronic density and of the angular dependences of $\gamma$-quanta created during the annihilation of positrons and electrons in carbon materials participating $s p^{2}$ or $s p^{3}$ hybridization [17]. Observation of the 1350 - and $1375-\mathrm{cm}^{-1}$ bands in diamond materials undoubtedly demonstrates the important contribution of $s p^{3}$ hybridization in their Raman spectra.

\section{Summary}

In this work, we demonstrated how changes of the structure of graphite materials lead to the processes of composition of phonon branches, which, in turn, bring to the existence of special vibrational states located out of the center of the Brillouin zone and appear in the IR and Raman spectra of such materials. This phenomenon, as well as the violation of strict selection rules for the carbon systems, offers new broader applications to optical spectroscopy techniques. The intensity of the bands forbidden under strict selection rules for the space symmetry group $D_{6 h}^{4}$ of graphite is nevertheless comparable to that of the allowed fundamental transitions and can be even stronger in some cases. Due to this fact, we could study, in detail, the shape of vibrational bands and reveal a doublet structure of diamond-like (D), additional (Ad), and graphite $(\mathrm{G})$ bands. We have investigated the intensity and the spectral position of these bands depending on the wavelength of the exciting laser radiation $\lambda_{L}$. For the first time, the two components of the $\mathrm{D}$ band at $1350 \mathrm{~cm}^{-1}$ and $1375 \mathrm{~cm}^{-1}$ are clearly observed. Our structural study of a large number of various structural modifications of disordered graphite allowed the observa- 
tion of the Raman intensity redistribution between these components depending on the degree of structural disorder. Doublet structures have been also observed for the Ad and G bands (1510-1540 $\mathrm{cm}^{-1}$ and 1590-1626 $\mathrm{cm}^{-1}$, respectively). Similar bands were previously reported by us and other authors in diamond-like films. The detailed study of the individual components of the doublets has been executed via application of a subtraction procedure to the original Raman spectra. We demonstrate how the interpretation of the bands is possible in view of the existence of a sharp edge of the bands and their one-side "smearing" under a reduction of the crystallite size. Useful information about the existing fine structure of the band was extracted here from the second derivative spectra. We have demonstrated that the observed doublet structure cannot always be explained in the framework of the CDR concept, which was previously successfully employed for the interpretation of doublet overtone vibrational states at $2697-2739 \mathrm{~cm}^{-1}$ registered for a single crystalline graphite. Some of the doublet components can be observed independently in various carbon materials and, moreover, may move in their spectral position under influence of some physical factors such as the ion implantation or the external pressure. These observations show the relationship of the doublet components to the structure of the materials under study and not to kinematic peculiarities of the Raman scattering. The theory of disordered condensed matter has else to be developed to include the consideration of such processes. Summarizing the experimental and theoretical results of Raman spectroscopy studies of carbon materials, we can conclude that the D and Ad bands are caused by various types of chemical bonding, including $s p^{2}$ and $s p^{3}$ hybridizations, in graphite materials (i.e., they are especially sensitive to intermediate structural states between a graphite-like and diamondlike coordination). As a consequence, the intensity of the band at $1350 \mathrm{~cm}^{-1}$ is to be higher, when the contribution of $s p^{2}$ hybridization is predominant. On the other hand, $s p^{3}$ hybridization prevails in the case of maximum intensity of the $1375 \mathrm{~cm}^{-1}$ band. In a similar fashion, the intermediate carbon structures determine the behavior of the additional Ad band. The studies carried out by us and the analysis of the extensive literary data have allowed the formulation of a new concept of phonon-like scattering of light applicable to a wide class of disordered condensed media. This not very common type of dispersion of light is specific for substances that are characterized with broad energy bands and poorly defined wave vector, rather than narrow dispersive branches $\omega(q)$. On contrary to a normal Raman scattering, where the frequencies of bands are fixed, the dependence of the positions and the intensities of vibrational bands on the experimental conditions (in particular, on the frequency of excitation radiation) is a typical feature of the phonon-like scattering processes. As we demonstrate in our study, carbon structures intermediate between graphite and diamond coordination (such as a range of metastable carbon structures formed under high pressures) with various contributions from $s p^{2}$ and $s p^{3}$ hybridizations make a significant contribution to the phonon-like Raman scattering. The intermediate states and the related phonon-like scattering of light are proven to be extremely sensitive to the conditions of the formation of carbon materials and to physical factors (ion implantation, thermal annealing, and radiation). Naturally, the intermediate structures of carbon possess specific electronic states, which may lead to specific resonance effects in Raman spectra observed by us and other authors. The effect reported here, involving the redistribution of the Raman intensity between the forbidden bands in the spectra of phonon-like scattering can be explained by the dependence of a residue of the zone structure on the structural modification of the matter. More studies both theoretical and experimental are needed to clarify the exact mechanisms of this phenomenon. The proposed concept and the obtained results may be useful for the investigation of metastable phases of amorphous carbon, scenarios of graphite-diamond transitions, and determination of the origin of high-temperature superconductivity.

1. M. Dresselhaus and G. Dresselhaus, in Light Scattering in Solids III. Recent Results, edited by M. Cardona and G. Guntherodt (Springer, Berlin, 1982), p. 8.

2. S. Reich and C. Thomsen, Phil. Trans. Royal. Soc. London A 362, 2271 (2004).

3. M.M. Sushchinskiy and Z.P. Mulhadmedov, Opt. Spectrosc. 16, 234 (1984); 9, 102001 (2010).

4. R.P. Vidano, D.B. Fishbach, and L.J. Willis, Solid State Commun. 39, 341 (1981).

5. A.V. Baranov, A.N. Bekhteev, Ya.S. Bobovich, and V.I. Petrov, Opt. Spectrosc. 62, 612 (1987).

6. M. Yoshikawa, G. Katagiri, H. Ishida, A. Ishitani, and T. Akamatsu, J. Appl. Phys. 64, 6464 (1988).

7. A.V. Baranov and E.A. Konshina, Opt. Spectrosc. 65, 856 (1983).

8. E. Fitzer and F. Pozploch, High Temper. High Press. 20, 449 (1988).

9. J. Wagner, M. Ramsteiner, Ch. Wild, and P. Koide, Phys. Rev. B 40, 1817 (1980). 
10. T. Tanabe, K. Niwase, and K. Nakamura, J. of Nucl. Mater. 168, 191 (1989).

11. B.S. Elman, M.S. Dresselhaus, G. Dresselhaus, E.W. Maby, and H. Mazurek, Phys. Rev. B 24, 1027 (1981).

12. B.S. Elman, M. Shayegan, M.S. Dresselhaus, H. Mazurek, and G. Dresselhaus, Phys. Rev. B 25, 4142 (1982).

13. A.V. Baranov, Ya.S. Bobovich, and V.I. Petrov, Opt. Specrosc. 63, 1026 (1987).

14. C. Thomsen and S. Reich, Phys. Rev. Lett. 85, 5214 (2000).

15. N.E. Korniyenko, A.P. Naumenko, A.G. Gontar, G.A. Ivankov, and G.A. Voronkin, in Proceed. of Intern. conference "Perspectives of Diamond Applications in Electronics" (Energoatomizdat, Moscow, 1991), p. 50.

16. R.J. Nemanich and S.A. Solin, Phys. Rev. B 20, 392 (1979).

17. F. Pazmigiani, E. Kay, and H. Seki, J. Appl. Phys. 64, 3031 (1988).

18. A.F. Goncharov, V.N. Denisov, B.N. Mavrin, V.B. Podobedov, Zh. Exper. Teor. Fiz. 94, 321 (1988).

19. Yu.A. Klyuyev, V.I. Neshpa, Yu.A. Dudenkov, S.D. Zvonyakov, and V.M. Zubkov, DAN SSSR 203, 1054 (1972).

20. I. Watanabe and K. Sugata, Japanese Journ. Appl. Phys. 27, 1808 (1988).

21. M.H. Brodsky, in Light Scattering in Solids I. Recent Results, edited by M. Cardona and G. Guntherodt (Springer, Berlin, 1979), p. 215.

22. A.F. Goncharov, I.N. Makarenko, and S.M. Stishov, J. Exp. Theor. Phys. 96, 670 (1989).

23. A.F. Goncharov, J. Exp. Theor. Phys. 98, 1824 (1990).
24. A.F. Goncharov, J. Exp. Theor. Phys. Lett. 51, 368 (1990).

Received 23.09.11

ФОНОНОПОДІБНЕ РОЗСІЯННЯ СВІТЛА

У ПОЛІКРИСТАЛІЧНИХ ВУГЛЕЦЕВИХ СТРУКТУРАХ

А.П. Науменко, М.Є. Корнієнко, В.М. Ящук,

В.М. Близнюк, С. Сінгаманені

$\mathrm{P}$ е $з$ ю м е

Розглянуто новий тип непружного розсіяння світла у невпорядкованих конденсованих середовищах, коли, на відміну від КР світла на коливаннях молекул та фононах з фіксованими коливальними частотами, відбувається зсув коливальних частот зі зміною частоти збудження. Це фононоподібне розсіяння світла притаманне проміжним станам упорядкування речовини із "замитою" зонною структурою при переході від молекул до кристалів, зокрема, для різноманітних вуглецевих структур, які $є$ проміжними між графітовою та алмазною координацією та містять деякі частки $s p^{2}$ - та $s p^{3}$-гібридизації. У дефектній (D) та додатковій (Ad) смугах чітко спостерігається дублетна структура та встановлений перерозподіл інтенсивностей між компонентами дублету 1350 і $1375 \mathrm{~cm}^{-1}$ для різних структурних модифікацій графіту. Виділення форм окремих складових компонент складних смуг здійснено у різницевих спектрах. Обговорюється природа дублетності коливальних смуг та обмеженість застосування концепції подвійного зв'язаного резонансу. Для встановлення природи аналізується асиметрія та тонка структура смуг, а також поведінка аналогічних смуг в алмазоподібних та вуглеводневих плівках та метастабільних фаз вуглецю при високих тисках. 